Check for updates

Cite this: RSC Adv., 2018, 8, 34168

\title{
In situ synchrotron XAS study of the decomposition kinetics of ZDDP triboreactive interfaces
}

\author{
Abdel Dorgham, (D) *a Pourya Parsaeian, ${ }^{a}$ Anne Neville, ${ }^{a}$ Konstantin Ignatyev, ${ }^{b}$ \\ Fred Mosselmans, (iD b Masabumi Masuko ${ }^{\mathrm{b}}$ and Ardian Morina ${ }^{\mathrm{a}}$
}

One of the major obstacles in replacing the widely used zinc dialkyldithiophosphate (ZDDP) antiwear additive with a more environmentally friendly one is the difficulty of time-resolving the surface species resulting from its decomposition mechanism under high contact pressure and temperature. To tackle this issue, a newly developed miniature pin-on-disc tribotester was coupled with synchrotron X-ray absorption spectroscopy (XAS) to perform in situ tribological tests while examining the composition of the formed triboreactive films. The results showed that in the case of bare steel surfaces the initial decomposition products are mainly zinc sulfate species, which with further shearing and heating are reduced to zinc sulfide mixed with metal oxides. The mixed base layer seems to enhance the tenacity of the subsequently formed zinc phosphate layers composing the main bulk of the protective triboreactive film. This base layer was not observed in the case of coated substrates with hydrogenated diamond-like carbon (a-C:H DLC) coating, which results in the formation of less durable films of small volume barely covering the contacting surfaces and readily removed by shear. Comprehensive decomposition pathways and kinetics for the ZDDP triboreactive films are proposed, which enable the control and modification of the ZDDP triboreactive films.

Received 4th June 2018

Accepted 22nd September 2018

DOI: $10.1039 / \mathrm{c} 8 \mathrm{ra0} 4753 \mathrm{~g}$

rsc.li/rsc-advances surfaces can be altered if the contacts are separated. This inevitable effect originates from four possible sources. First, the adventitious entities such as carbon can adsorb from the ambient atmosphere to the contact area. ${ }^{8}$ In such a case, the presence of carbon can either misrepresent the products of the decomposition reaction or add a layer that impedes or at least attenuates the signal coming from the layers beneath when probed by one of the surface-sensitive analysis techniques such as XPS. ${ }^{9}$ Second, water from the relative humidity of the ambient atmosphere can react with the separated contacting surfaces and possibly with the formed tribofilm, and thus alters the nature of the tribofilm, e.g. can produce sulfate ${ }^{10-12}$ or cleave the phosphate chains composing the tribofilm. ${ }^{11,13}$ Third, probing the contacting surfaces after the test can conceal the formation and kinetics of any intermediate species during the initial stage of the decomposition reaction. ${ }^{14}$ Fourth, during sliding at high temperature and under high contact pressure, triboemission from the substrate ${ }^{15}$ availability of cations, ${ }^{16}$ heat and shear ${ }^{17}$ make a continuous environment for chemical reactions to evolve, which cannot be captured completely using only ex situ technique. The four sources combined can partially obscure the tribochemical reactions from the first stages of the additive decomposition in the oil to the formation of the last layer of the protective tribofilm on the rubbed areas.

In order to overcome the aforementioned limitations, recently a new in situ tribotester has been developed and coupled with X-ray absorption spectroscopy (XAS) to obtain real
${ }^{a}$ Institute of Functional Surfaces, School of Mechanical Engineering, University of Leeds, Leeds LS2 9JT, UK. E-mail: a.dorgham@Leeds.ac.uk

${ }^{b}$ Diamond Light Source Ltd., Diamond House, Harwell Science and Innovation Campus, Didcot, Oxfordshire OX11 ODE, UK

${ }^{c}$ Department of Chemical Engineering, Tokyo Institute of Technology, Tokyo, Japan 
time surface analysis information. ${ }^{14}$ Using this technique, it was possible to follow the evolution of the $\mathrm{P}$ and $\mathrm{S}$ species composing the tribofilm over time. It was found that initially short phosphate chains are formed, which under rubbing are polymerized to form longer chains. However, this view is in contrast with some previous $e x$ situ studies ${ }^{18-21}$ suggesting that initially long chains are formed, which for various reasons can be depolymerized into shorter chains. The depolymerization was suggested to occur due to the high shear stress at the asperity contacts, which can possibly cleave the long phosphate chains into shorter ones, and to wear that can remove the weakly adhered long phosphate chains from the surface. ${ }^{21}$ In addition, the presence of iron oxide especially near the metal surface can depolymerize the long phosphate chains to form short Fe- or mixed Fe-Zn phosphates. ${ }^{18-20}$

Similar to the phosphorus species, there is also no clear consensus on the evolution of the sulfur species found in the ZDDP tribofilm. The vast majority of the previous ex situ studies suggested that sulfur exists in the tribofilm in the reduced sulfide form especially near the metal surface. ${ }^{16,19,22,23}$ However, other studies ${ }^{10,23-26}$ showed that in the beginning of rubbing, sulfur appears in the oxidized sulfate form, which under rubbing seems to be reduced to sulfide. This is in agreement with our previous in situ results using the newly developed in situ tribotester. ${ }^{14}$

The discrepancy in the previous data might be related to the applied conditions of temperature, contact pressure, rubbing time and availability of metallic cations such as Fe and $\mathrm{Zn}$. To examine the evolution of the tribofilm in more details, this study aims at following the decomposition reaction in situ using a range of operating conditions with much emphasis on the changes in the beginning of the decomposition reaction. This will enable us to capture the formation of any intermediate species leading to a better understanding of the decomposition of the ZDDP and formation of its protective tribofilm.

\section{Materials and methods}

\section{Materials and operating conditions}

The lubricant used in this study is poly- $\alpha$-olefin (PAO) based oil containing secondary ZDDP antiwear additive $(0.08 \mathrm{wt} \% \mathrm{P}$ concentration) and a trace of ester oil to aid the solubility of ZDDP in PAO. The PAO oil and ZDDP were obtained from Afton Chemical, UK, and used as received. The oil has a kinematic viscosity of $18.7 \mathrm{cSt}$ at $40{ }^{\circ} \mathrm{C}$ and $4.2 \mathrm{cSt}$ at $100{ }^{\circ} \mathrm{C}$. For all the tests reported in this study, a small amount of $2 \mathrm{ml}$ of the oil was introduced to the counterbodies such that the disc and ball are fully lubricated, i.e. about $0.5 \mathrm{~mm}$ of the ball height was immersed in the oil.

The counterbodies consist of a rotating disc and stationary ball. In order to obtain a clear wear scar on the disc for its easy identification during the in situ XAS tests, the disc was selected to have large dimensions of 110 and $85 \mathrm{~mm}$ for its outer and inner diameters, respectively, and to be made of AISI 1074 steel of low hardness of 47 on Rockwell scale compared to the ball of average hardness of 64 on Rockwell scale, which is made of AISI 52100 steel and has $5.5 \mathrm{~mm}$ diameter. To examine the role of temperature and contact pressure on the decomposition of ZDDP, two temperatures $\left(80\right.$ and $\left.25^{\circ} \mathrm{C}\right)$ and two contact pressures (1.0 and 2.2 GPa) were tested. These were selected such that they simulate the cold start and normal operating conditions of the internal combustion engine of passenger cars during mild and extreme loadings. The experimental conditions during the in situ XAS experiments are summarized in Table 1. The sliding speed for all the listed tests was fixed at $50 \mathrm{rpm}$.

In order to examine the role of metallic cations in the decomposition reaction of the additive, one experiment was performed using coated discs and balls with a multilayer coating of hydrogenated diamond-like carbon (a-C:H DLC). The coating consists of a $\mathrm{Cr}$ base layer and an a-C:H top layer. In between these two layers, a graded interlayer of WC was deposited in order to improve adhesion and grading of the mechanical properties between the two materials. The coating was prepared using a hybrid magnetron sputtering and plasma enhanced chemical vapor deposition (PECVD). The deposition temperature during the entire process was lower than $200^{\circ} \mathrm{C}$. At first, prior to any layer coating, a Plasma Surface Engineering (PSE) step was used to etch and clean the substrate using a bias etching voltage of $-200 \mathrm{~V}$ through a $\mathrm{W}$ filament plasma source for $45 \mathrm{~min}$. Afterwards, the $\mathrm{Cr}$ coating layer was deposited using reactive magnetron sputtering for 25 minutes without surface bias. Immediately after the previous step stopped, acetylene gas was introduced to begin the a-C:H layer deposition using PECVD for 150 minutes using a surface bias of $-780 \mathrm{~V}$. This procedure resulted in an overall coating thickness of $2 \pm 0.5 \mu \mathrm{m}$ with a hardness of about 70 on Rockwell scale.

\section{In situ XAS tribological rig}

The tribological tests were performed using a newly developed rig, which was designed to function as a pin-on-disc apparatus.

Table 1 List of the experimental conditions during the in situ XAS measurements

\begin{tabular}{llllll}
\hline$\#$ & Counter surfaces & Additive & $T\left({ }^{\circ} \mathrm{C}\right)$ & $P(\mathrm{GPa})$ & Duration \\
\hline 1 & Steel ball/steel disc & ZDDP & 80 & $-^{a}$ & $0^{b}, 1,5,15,20,24 \mathrm{~h}$ \\
2 & Steel ball/steel disc & ZDDP & 80 & 2.2 & $0^{b}, 2.5,10,20,30,60 \mathrm{~min}$ \\
3 & Steel ball/steel disc & ZDDP & 80 & 1.0 & $0^{b}, 6 \mathrm{~s}, 30 \mathrm{~s}, 1 \mathrm{~min}, 2.5 \mathrm{~min}, 5 \mathrm{~min}$, \\
4 & Steel ball/steel disc & ZDDP & 25 & 1.0 & $10 \mathrm{~min}, 20 \mathrm{~min}$ \\
5 & DLC ball/DLC disc & ZDDP & 80 & 1.0 &
\end{tabular}

${ }^{a}$ Thermal film with no applied contact pressure. ${ }^{b}$ Before the start of heating for thermal films or rubbing for tribofilms. 
(a)

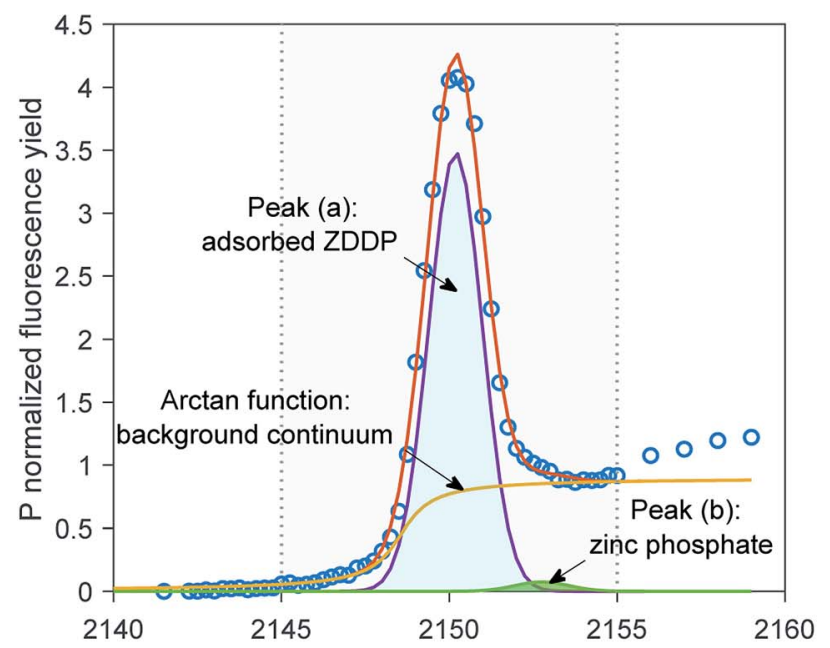

(b)

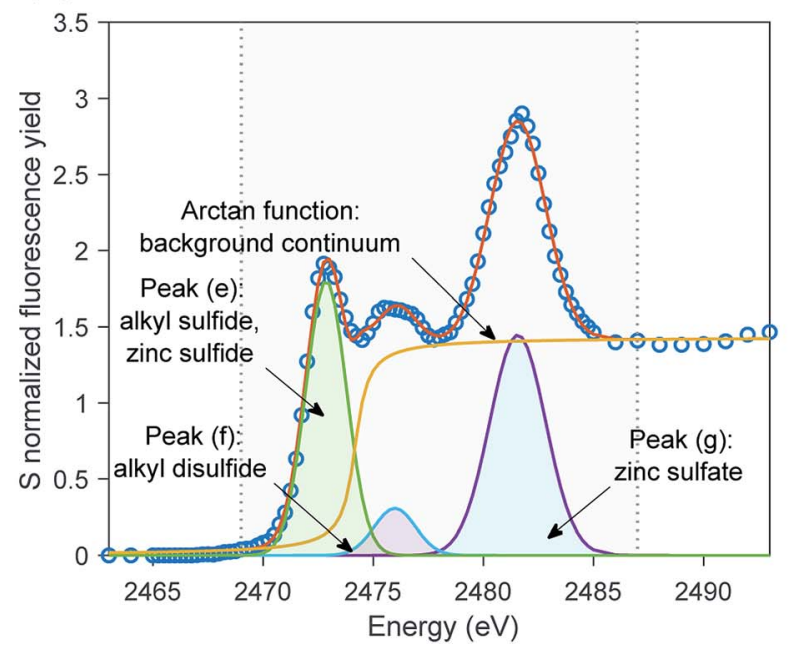

Fig. 1 Example of the peak fitting used to determine the areas of the peaks forming (a) $\mathrm{P}$ and (b) $\mathrm{S}$ k-edges respectively. The example is based on the signal of ZDDP thermal film generated after $5 \mathrm{~h}$ of heating time at $80{ }^{\circ} \mathrm{C}$. The energy range framed by the grey dotted lines represents the fitting window of the $\mathrm{P}$ and $\mathrm{S}$ peaks.

Details of this rig were published elsewhere. ${ }^{14}$ During the tribological tests, the disc was rotating around the motor shaft at a controlled speed while the pin represented by a $5.5 \mathrm{~mm}$ diameter ball was stationary.

\section{XAS beamline and detector setup}

The XAS incident beam source was I18 microfocus spectroscopy beamline located in the Diamond Light Source facility - Oxford (UK). ${ }^{27}$ Four-element Vortex-ME4 silicon drift detector (Hitachi, USA) with Xspress3 electronics processing (Quantum Detectors, UK) was used to in situ acquire the fluorescence yield (FY) spectra at the $\mathrm{P}$ and $\mathrm{S}$ k-edges of the formed tribofilms over time. The incident beam was at an angle $2^{\circ}$ relative to the disc surface. The beam size was $50 \times 50 \mu \mathrm{m}^{2}$ resulting in a footprint of $50 \times 1433 \mu \mathrm{m}^{2}$ on the disc. The large disc dimensions that produce a large wear scar diameter can accommodate a beam footprint length up to $6.2 \mathrm{~mm}$. The detector was placed at a right angle with the incident source at a $20 \mathrm{~cm}$ distance above the analyzed sample. The implications of this vertical geometry including the possible attenuation of the peaks' heights were discussed in detail elsewhere. ${ }^{14,28}$

The XAS spectra of $\mathrm{P}$ and $\mathrm{S}$ k-edges were acquired in two locations. i.e. one inside and one outside the wear scar. The wear scar on the disc was located using scanning micro-X-ray fluorescence (XRF) mapping. The pre-edge region was scanned using $3 \mathrm{eV}$ energy step and acquisition time of $0.5 \mathrm{~s}$. The Xray Absorption Near Edge Structure (XANES) edge region was scanned using $1 \mathrm{eV}$ energy step and acquisition time of $0.5 \mathrm{~s}$. The Extended X-ray Absorption Fine Structure (EXAFS) postedge region was scanned using a high resolution energy step of $0.1 \mathrm{eV}$ and long acquisition time of $3 \mathrm{~s}$. A good signal to noise ratio was obtained using a single scan only. To insure no radiation damage occurs, few scans were repeated three times at the same position on the disc without observing any changes from scan to scan. Athena software (version 0.9.24) was used to correct the background and normalize the acquired data following standard procedures suggested by Ravel and Newville. ${ }^{29}$ Using this software, the qualitative changes in the XANES $\mathrm{P}$ and $\mathrm{S}$ k-edge spectra were quantified by fitting the peaks of the k-edge. An example of the peak fitting used to determine the area of the adsorbed ZDDP and phosphate peaks at the P k-edge and the areas of peaks (e) and (f) of the sulfides and peak (g) of sulfate at the $\mathrm{S}$ k-edge is shown in Fig. 1 for ZDDP thermal film generated after 5 hours of heating. The fit used Gaussian peaks and an arctan function to remove the background continuum. ${ }^{30}$ It should be noted that peak (f) appears to consist of multiple peaks, i.e. could be two or more. However, this is a very small peak that fitting it with multiple peaks would not be accurate as no information is available in the literature for identifying such peaks. Thus, peak (f) was fitted with a single peak for qualitatively stating when it appears or disappears and how it evolves. The standard deviation of the positions of the XANES P and S kedge peaks was reported based on the mean across the different experiments.

\section{Wear measurement}

NPLEX white light interferometer (Bruker, USA) was used to obtain 3D images of the worn samples after the in situ tests in order to quantify the wear scar width and depth. The collected interference pattern was analyzed using Vision64 software (Bruker, USA). The wear was then quantified by measuring the width and depth of the wear scar at different locations. All the reported wear measurements in this work are based on the disc because of its lower hardness than the ball countersurface and thus its higher susceptibility to wear. As the presence of any tribofilm on the surface can affect the refractive index and thus the interference pattern, the wear measurements were performed after rinsing the tribological surfaces in heptane and then removing the tribofilm using a droplet of $0.05 \mathrm{M}$ Ethylenediaminetetraacetic acid (EDTA) solution in distilled water placed on the wear scar for at least $60 \mathrm{s.}^{31,32}$ 


\section{Results and discussion}

\section{Friction and wear performance}

The evolution of the friction coefficient over time using plain and DLC coated countersurfaces rubbed at 25 and $80{ }^{\circ} \mathrm{C}$ are shown in Fig. 2a. In the case of plain hardened spring steel surfaces rubbed at high temperature, the friction coefficient after the running-in period was at its maximum value around 0.12. On the other hand, in the case of the surfaces rubbed at low temperature or surfaces were coated with H-DLC, the friction coefficient was lower at 0.09. Another interesting feature is the difference in the running-in period between the tests. The plain steel countersurfaces rubbed at high temperature showed a relatively large change in the friction coefficient from initially about 0.10 to 0.12 , whereas for the other cases, the friction coefficient changed only slightly.

The evolution of the friction coefficient can be related to the presence or absence of the ZDDP tribofilm on the contacting
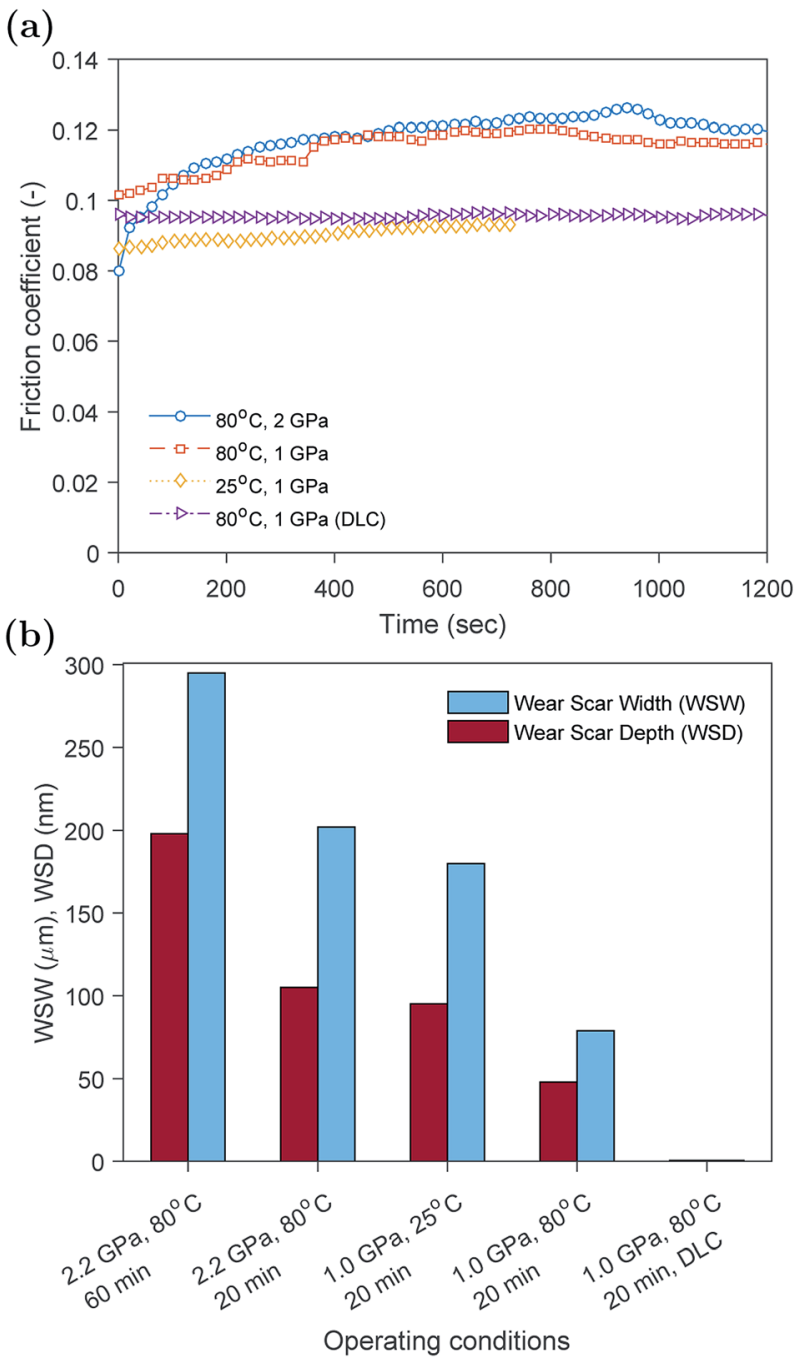

Fig. 2 (a) Evolution of the friction coefficient during the in situ tests using plain steel and DLC coated countersurfaces, and (b) wear scar width and depth measured using interferometry technique after removing the tribofilm using EDTA. surfaces. Previous studies ${ }^{33-35}$ showed that the formation of ZDDP tribofilms causes an increase in the friction coefficient. This was related to either the ability of the tribofilm to hinder the oil entrainment between contacting surfaces or the increased roughness of the formed tribofilm. The inability to form a tribofilm at low temperature is expected as the formation rate under such conditions can be extremely slow. ${ }^{36}$ This is because of the thermal decomposition nature of the ZDDP additive, which requires high temperature to activate the reaction of the additive in the oil and consequently the formation of a protective tribofilm on the contacting surfaces.

The friction results of the surfaces coated with non-doped $\mathrm{H}$ DLC seem to suggest that no tribofilm was formed on the surface, which is supported by the similar friction behavior to the case of plain countersurfaces rubbed at low temperature. The absence of any formed tribofilm on non-doped H-DLC was reported extensively in the literature, ${ }^{37-42}$ whereas other studies found that some thin tribofilms can be formed. ${ }^{43-49}$ The formation and composition of such films will be investigated further using the in situ surface analysis of XANES P and S kedges, which is discussed in detail in the subsequent sections.

The wear results obtained using white light interferometry are shown in Fig. 2b. The width and depth of the wear scar seem to decrease with decreasing the load and rubbing time or increasing the temperature. This is expected as the less harsh the operating conditions become the less the wear would result. The beneficial effect of temperature on wear is related to the formation of a protective tribofilm on the contacting asperities at high temperature that helps protect the rubbing surfaces. However, at low temperature this tribofilm cannot be formed, which results in higher wear.

In the case of DLC coated countersurfaces, no wear was detected. In addition, no visible wear track or tribofilm was discernible.

\section{Peak identification at $P$ and $S$ k-edges}

The evolutions of the normalized $\mathrm{P}$ k-edge spectra formed after different heating and shearing times are shown in Fig. 3a-7a. The identifications of the main and secondary peaks of these spectra are summarized in Table 2 . The $\mathrm{P}$ k-edge spectra consist primarily of one pre-edge peak and four main peaks. The preedge peak $\left(\mathrm{a}^{\prime}\right)$ was not observed at $2148.0 \pm 0.1 \mathrm{eV}$ as a distinctive peak. The peak is mainly related to iron phosphate. ${ }^{50}$ The absence of this peak indicates that the formed phosphate contains mainly zinc rather than iron or that iron phosphate has low concentration or form localized isolated patches within the tribofilm. Peak (a) appears at $2150.2 \pm 0.2 \mathrm{eV}$ even before heating or rubbing started and hence it can be related to ZDDP. This ZDDP can be in the form of unreacted molecules or adsorbed molecules transformed into a rearranged linkage isomer in which the alkyl groups have migrated from $\mathrm{O}$ to $\mathrm{S}$ atoms. ${ }^{51,52}$ Peak (b) starts to distinctively appear at $2152.5 \pm$ $0.2 \mathrm{eV}$ as heating or rubbing progresses. The peak can be attributed to the decomposition of ZDDP and formation of zinc phosphate whether of short or long chains. ${ }^{\mathbf{1 0 , 1 4 , 5 3 , 5 4}}$ Two postedge peaks (c) and (d) appear at $2159.0 \pm 0.3 \mathrm{eV}$ and $2169.5 \pm$ 
(a)

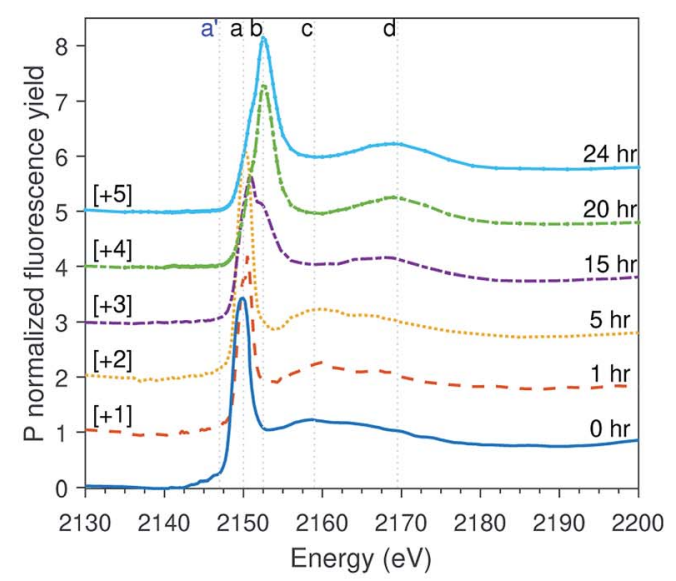

(b)

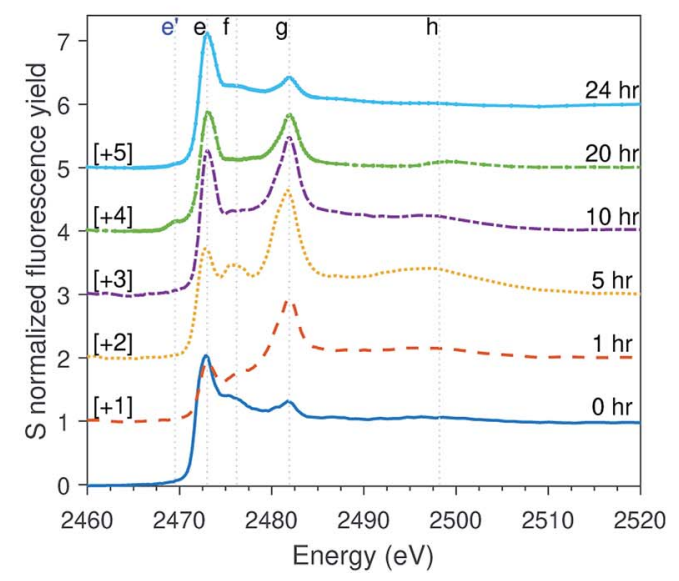

(c)

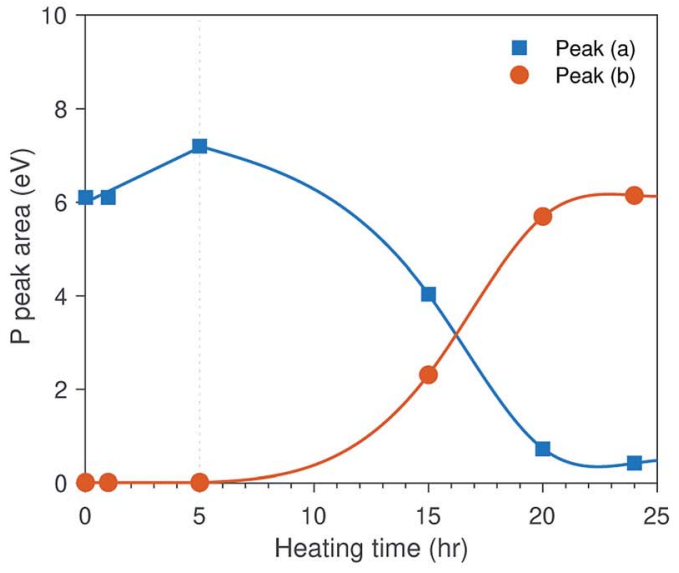

(d)

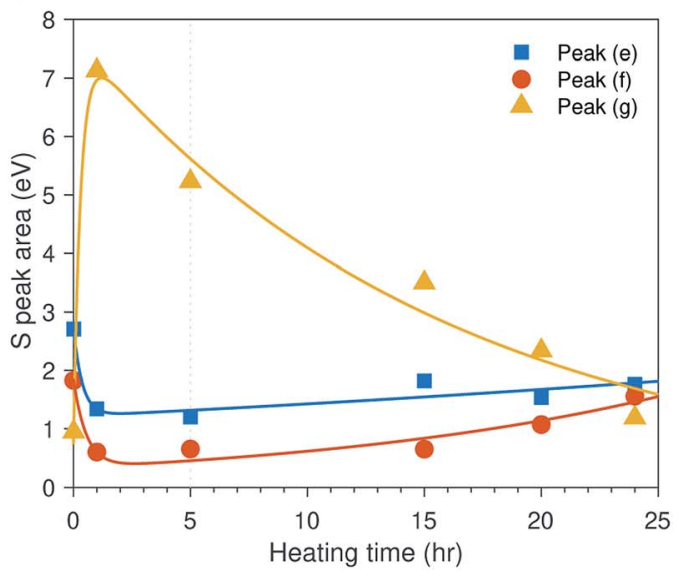

Fig. 3 Spectra evolution of the ZDDP normalized fluorescence yield after different heating times at $80^{\circ} \mathrm{C}$ without shear. (a) and (b) Spectra of $\mathrm{P}$ and S k-edges, respectively. (c) P k-edge normalized heights of peak (a): adsorbed ZDDP and peak (b): zinc phosphate. (d) S k-edge normalized heights of peak (e): alkyl sulfide and zinc sulfide, peak (f): alkyl disulfide and peak (g): zinc sulfate. Each curve in (a) and (b) is vertically offset for clarity by adding a constant, as specified on each curve, relative to the first curve.

$0.2 \mathrm{eV}$, respectively. Post-edge peaks originate from the multiple scattering resonance of the photo-electrons around atoms neighboring the excited atom. Therefore, they can provide information of the local electronic structure and thus the composition of the formed tribofilm. For instance, peak (c) was only observed before heating or shearing has begun, i.e. in the unreacted or adsorbed ZDDP spectrum, and after short rubbing time during which the unreacted or adsorbed ZDDP dominated the XANES spectrum. This indicates that this peak can be related to unreacted or adsorbed ZDDP. As rubbing or heating continued, more of the unreacted or adsorbed ZDDP was decomposed to form zinc phosphate. This caused peak (c) to vanish and peak (d) to become more visible after long rubbing or heating time. This indicates that peak (d) can be related to the formation of phosphates, i.e. mainly zinc phosphate. The high energy peaks (c) and (d) in the post-edge EXAFS region can only be fully analyzed when the data are fitted to theoretical models to deduce structural information. ${ }^{55}$ This was not performed in this study as it requires extended spectra with high signal-to-noise ratio.
The evolutions of the normalized S k-edge spectra formed after different heating and shearing times, which are shown in Fig. 3b-7b, indicate that the spectra consist primarily of one pre-edge peak and four main peaks. The pre-edge peak $\left(\mathrm{e}^{\prime}\right)$ appears at $2469.5 \pm 0.1 \mathrm{eV}$, which can be related to the presence of $\mathrm{FeS}^{56}$ or alkyl sulfide. ${ }^{54}$ Peak, (e) was observed at $2472.7 \pm$ $0.2 \mathrm{eV}$, which can be related to $\mathrm{ZnS}^{53}$ or alkyl sulfide $\mathrm{e}^{53,54,56}$ from the unreacted or adsorbed ZDDP molecules. The initial decrease and then increase in the intensity of peak (e) over heating or rubbing time suggest that initially peak (e) can be attributed mainly to alkyl sulfide, whereas over time it is converted into zinc sulfide ( $\mathrm{ZnS}$ ). Thus, after the initial stage of heating or rubbing, peak (e) can be assigned to a mixture of alkyl sulfide and ZnS, which evolves over heating or rubbing time such that the concentration of alkyl sulfide is continuously decreasing whereas the one of $\mathrm{ZnS}$ is continuously increasing. Peak (f), which appears at $2476.0 \pm 0.3 \mathrm{eV}$, indicates the presence of alkyl disulfide from the unreacted or adsorbed ZDDP. ${ }^{54}$ As alkyl sulfide, peak (e), and alkyl disulfide, peak (f), appear in the spectra, this indicates that the detected ZDDP is not in the 
Table 2 Peak positions of $\mathrm{P}$ and $\mathrm{S}$ k-edges (FY) spectra of the triboand thermal films of $\mathrm{P}$-based additives

\begin{tabular}{|c|c|c|c|}
\hline Peak & Position $(\mathrm{eV})$ & Assignment & Reference \\
\hline \multicolumn{4}{|c|}{ P k-edge peaks } \\
\hline $\mathrm{a}^{\prime}$ & $2148.0 \pm 0.1$ & Fe phosphate & 50 \\
\hline a & $2150.2 \pm 0.2$ & $\mathrm{ZDDP}(-\mathrm{P}-\mathrm{S}-)$ & 14,53 and 54 \\
\hline b & $2152.5 \pm 0.2$ & Zn phosphate & $10,14,53$ and 5 \\
\hline$c^{a}$ & $2159.0 \pm 0.3$ & $\mathrm{ZDDP}(-\mathrm{P}-\mathrm{S}-)$ & \\
\hline $\mathrm{d}^{a}$ & $2169.5 \pm 0.2$ & Zn phosphate & \\
\hline \multicolumn{4}{|c|}{$S$ k-edge peaks } \\
\hline \multirow[t]{2}{*}{$\mathrm{e}^{\prime}$} & $2469.5 \pm 0.1$ & $\mathrm{FeS}$ & 56 \\
\hline & & $\mathrm{ZDDP}(-\mathrm{S}-\mathrm{R})$ & 54 \\
\hline \multirow[t]{2}{*}{ e } & $2472.7 \pm 0.2$ & $\mathrm{ZnS}$ & 53 \\
\hline & & ZDDP $(-S-R)$ & 53,54 and 56 \\
\hline $\mathrm{f}$ & $2476.0 \pm 0.3$ & ZDDP (-S-S-R) & 54 \\
\hline \multirow[t]{2}{*}{$\mathrm{g}$} & $2481.8 \pm 0.2$ & $\mathrm{ZnSO}_{4}$ & 57 \\
\hline & & $\mathrm{FeSO}_{4}$ & 54 and 56 \\
\hline $\mathrm{h}^{a}$ & $2498.2 \pm 0.3$ & $-\mathrm{SO}_{4}$ & 58 \\
\hline
\end{tabular}

${ }^{a}$ Broad peak due to multiple scattering resonance of the photoelectrons.

form of unreacted molecules but instead in the form of adsorbed ZDDP molecules transformed into a rearranged linkage isomer in which the alkyl groups have migrated from $\mathrm{O}$ to $\mathrm{S}$ atoms. ${ }^{51,52}$ Peak (g), which appears at $2481.8 \pm 0.2 \mathrm{eV}$, is a fingerprint of $\mathrm{ZnSO}_{4}$ (ref. 57) or $\mathrm{FeS}_{4} \cdot{ }^{54,56}$ As no pre-edge peak $\left(\mathrm{e}^{\prime}\right)$ appears in the different spectra over time, which is a fingerprint of iron, peak (g) can be mainly assigned to $\mathrm{ZnSO}_{4}$. The presence of sulfate species, whether $\mathrm{FeSO}_{4}$ or $\mathrm{ZnSO}_{4}$, can also be indicated by peak (h), which appears in the high energy post-edge region at $2498.2 \pm 0.3 \mathrm{eV}$. For instance, this peak does not appear in the adsorbed ZDDP spectrum or after short heating time during which the adsorbed ZDDP dominates the XANES spectrum. As more sulfate is formed with progressive heating or rubbing, peak (h) starts to appear and become visible.

\section{ZDDP thermal films formed at $80{ }^{\circ} \mathrm{C}$}

The evolution of the areas of the peaks observed at the $\mathrm{P}$ and $\mathrm{S} \mathrm{k}$ edges after different heating times is shown in Fig. 3c and d, respectively. During the initial stage of heating, the area of the adsorbed ZDDP, peak (a), appeared to increase over time whereas the area of the zinc phosphate, peak (b), was negligible indicating that no phosphate chains were formed. The evolution of sulfur in the same early stage suggests that initially the alkyl sulfide, peak (e), and alkyl disulfide, peak (f), from the adsorbed ZDDP start to decompose rapidly to form zinc sulfate, peak $(\mathrm{g})$. This is in line with previous reports, ${ }^{\mathbf{1 4 , 5 9}}$ which suggest that during the ZDDP thermal decomposition the sulfate concentration increases with heating while sulfide decreases possibly due to its reaction with the substrate covered with oxides leading to the oxidation of the sulfur species into sulfates.

After the initial phase, the area of the adsorbed ZDDP starts to decrease exponentially over heating time before reaching steady state. In contrast, the zinc phosphate peak showed an opposite trend of increasing in area exponentially, but with a similar rate, before reaching steady state. This possibly indicates a continuous consumption of the adsorbed ZDDP to form phosphate chains of increasing length over heating time. In parallel, the progressive change of sulfur over time suggests a gradual evolution in the composition of the thermal film matrix favoring the initial formation of zinc sulfate, which are eventually reduced into more stable zinc sulfide. This is in line with the results of several previous studies ${ }^{\mathbf{1 0 , 1 6 , 1 9 , 2 0 , 6 0}}$ suggesting that the ZDDP additive forms mainly sulfide species in the final stages of the decomposition reaction.

The initial changes in the adsorbed ZDDP as revealed by XANES surface analysis, which show a delay between the adsorption of ZDDP and the formation of zinc phosphate, indicate the presence of an initial induction period. This is the first direct evidence of the possible presence of this period preceding the formation of the zinc phosphate chains composing the main bulk of the triboreactive film. The duration of the induction period can last for hours in the case of thermal films whereas it is shortened to only a couple of minutes or even seconds in the case of tribofilms formed under high temperature and contact pressure, as discussed in the following sections. This confirms that rubbing can have a potent effect on accelerating the ZDDP decomposition and its tribofilm formation.

\section{ZDDP tribofilms formed at $80{ }^{\circ} \mathrm{C}$ and $2.2 \mathrm{GPa}$}

The evolution of the areas of the $\mathrm{P}$ and $\mathrm{S}$ peaks of the ZDDP tribofilms formed at $80^{\circ} \mathrm{C}$ and $2.2 \mathrm{GPa}$ is shown in Fig. $4 \mathrm{c}$ and d, respectively. Similar to the case of the thermal films, the areas of the $\mathrm{P}$ and $\mathrm{S}$ peaks appear to undergo two distinctive phases. During the first phase, the area of the adsorbed ZDDP, peak (a), decreased sharply whereas the area of the zinc phosphate, peak (b), increased with a similar rate. The results suggest that the ZDDP molecules adsorb preferentially to the steel surface during the heating time and are ready to form the tribofilm consisting mainly of zinc phosphate immediately once shearing commences. Furthermore, during the initial stage of shearing, the area of zinc sulfate, peak (g), appeared to increase sharply over time. In contrast, the areas of alkyl sulfide, peak (e), and alkyl disulfide, peak (f), appeared to decrease over the same period with a relatively similar fast rate.

As rubbing continued, i.e. during the second phase, the area of the zinc sulfate, peak (g), decreased gradually over shearing time. In contrast, the areas of alkyl sulfide and zinc sulfide, peak (e), and alkyl disulfide, peak (f), showed an opposite trend of increasing in area, but with a relatively similar rate. The comparable rates can possibly indicate a continuous consumption of the zinc sulfate to form more stable zinc sulfide. Furthermore, the area of the adsorbed ZDDP peak appeared to be steady and of minuscule amount throughout the tribotest with a negligible decrease. Similarly, the area of the zinc phosphate peak appeared to be steady throughout the test with a mild decrease over shearing time. The decrease in the area of the zinc phosphate peak can be related to the scission of 
(a)

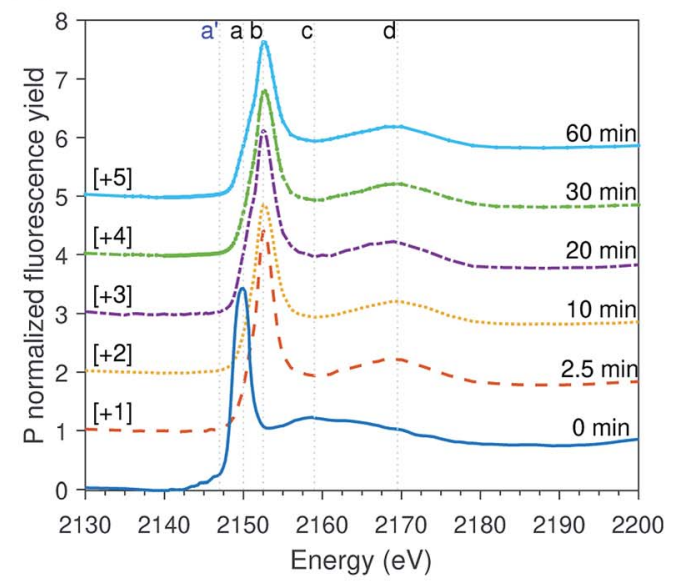

(b)

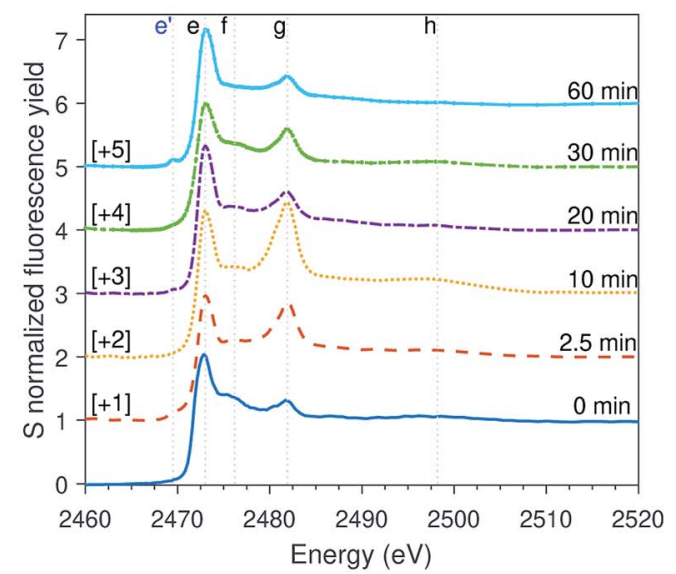

(c)

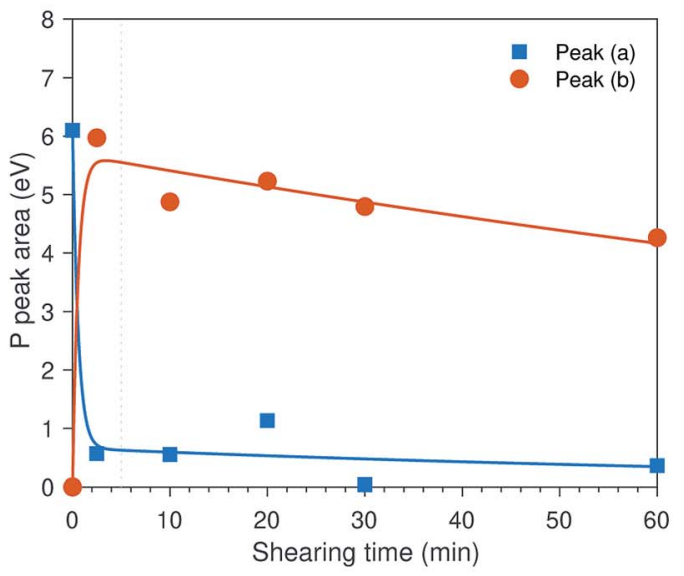

(d)

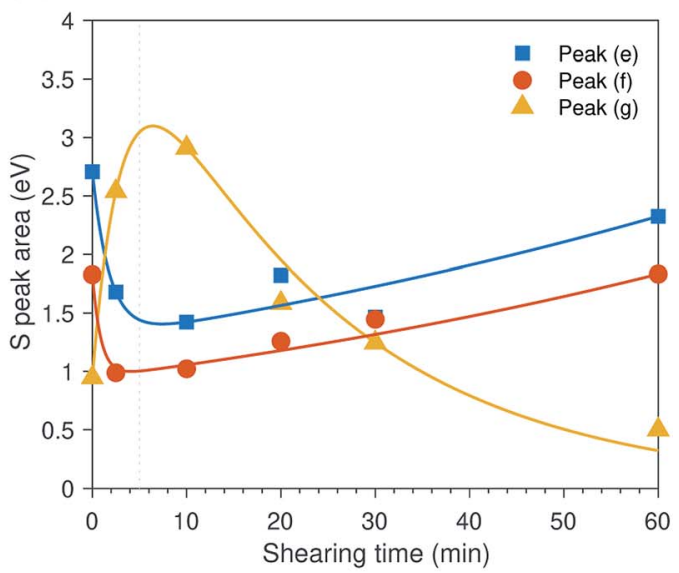

Fig. 4 Spectra evolution of the ZDDP normalized fluorescence yield after different shearing times at $80^{\circ} \mathrm{C}$ and $2.2 \mathrm{GPa}$. (a) and (b) Spectra of $\mathrm{P}$ and S k-edges, respectively. (c) P k-edge normalized heights of peak (a): adsorbed ZDDP and peak (b): zinc phosphate. (d) S k-edge normalized heights of peak (e): alkyl sulfide and zinc sulfide, peak (f): alkyl disulfide and peak (g): zinc sulfate. Each curve in (a) and (b) is vertically offset for clarity by adding a constant, as specified on each curve, relative to the first curve.

long phosphate chains into shorter ones under the large contact pressure and to severe surface wear that can remove the long chains.

\section{ZDDP tribofilms formed at $80{ }^{\circ} \mathrm{C}$ and $1.0 \mathrm{GPa}$}

The evolution of the areas of the peaks observed at the $\mathrm{P}$ and $\mathrm{S} \mathrm{k-}$ edges, respectively, after different rubbing times are shown in Fig. $5 \mathrm{c}$ and $\mathrm{d}$. The initial inspection of the data indicates that the changes in the tribofilm spectra over rubbing time are comparable to the ones of the tribo- and thermal films discussed in the previous sections. However, the spectra formed at 1.0 GPa seem to evolve at a faster rate than the ones of the thermal films and slower than the ones of the tribofilms formed at larger contact pressure of $2.2 \mathrm{GPa}$. Moreover, contrary to the slowly evolving thermal films and similar to the tribofilms formed under high contact pressure of $2.2 \mathrm{GPa}$, the tribofilms formed at 1.0 GPa do not appear to have an initial induction period in which the ZDDP molecules just adsorb to surface without any zinc phosphate formation. Shearing the interface between the contacting surfaces seems to vastly shorten the induction period, which highlights its role in accelerating the additive decomposition kinetics.

The evolution of the S k-edge spectra of the ZDDP tribofilms presented in Fig. 5d shows a similar trend to the one observed in the spectra of the tribo- and thermal films discussed before. The only difference is that with the progression of shearing, the change in the areas sulfides, i.e. alkyl sulfide and zinc sulfide: peak (e) and alkyl disulfide: peak (f), and sulfate, i.e. zinc sulfate: peak $(\mathrm{g})$, seems to be negligible. The constant concentrations instead of the reduction of zinc sulfate to zinc sulfide may be a result of the relatively less harsh operating conditions as compared to the tribofilm formed at higher contact pressure of $2.2 \mathrm{GPa}$. In addition, the reduction of sulfate seems to require longer time than the short period of 20 minutes of the tribotest.

\section{ZDDP tribofilms formed at $25{ }^{\circ} \mathrm{C}$ and $1.0 \mathrm{GPa}$}

The evolution of $\mathrm{P}$ and $\mathrm{S}$ k-edge spectra shown in Fig. 6 indicates that contrary to the slowly evolving thermal film and similar to the previously studied fast evolving tribofilms formed at high temperature, the tribofilm formed at low temperature 
(a)

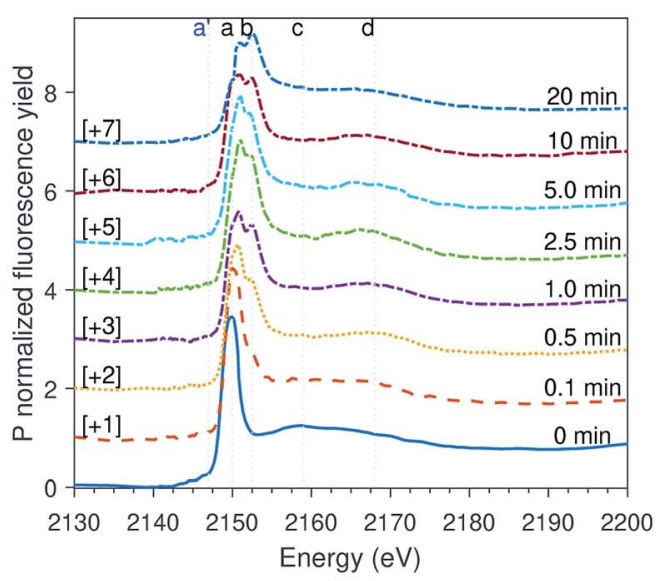

(b)

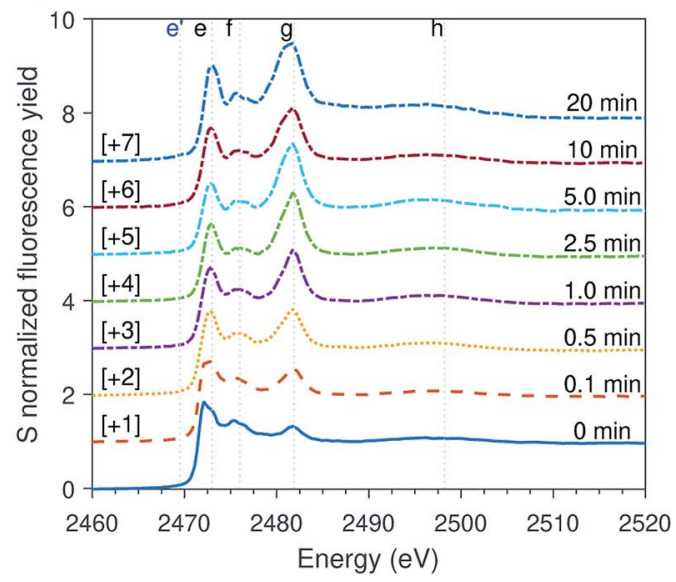

(c)

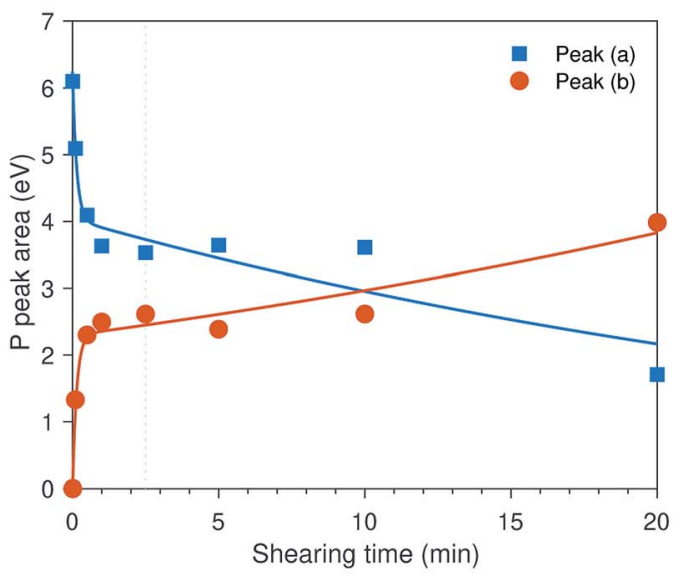

(d)

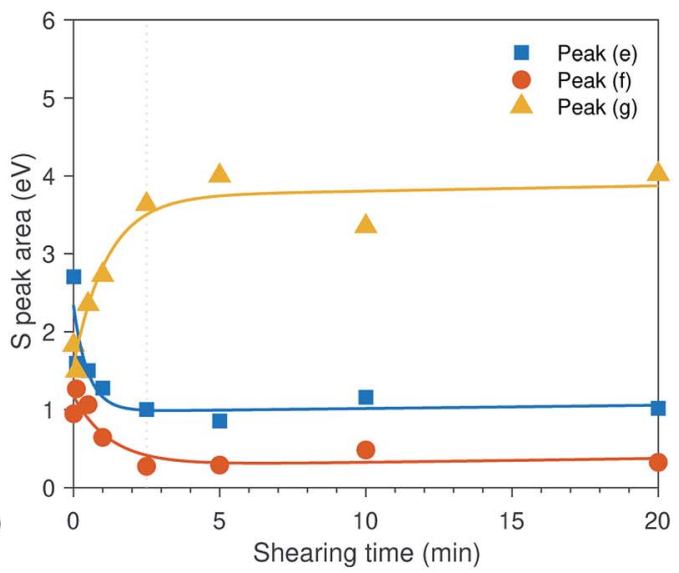

Fig. 5 Spectra evolution of the ZDDP normalized fluorescence yield after different shearing times at $80^{\circ} \mathrm{C}$ and $1 \mathrm{GPa}$. (a) and (b) Spectra of $\mathrm{P}$ and $\mathrm{S}$ k-edges, respectively. (c) P k-edge normalized heights of peak (a): adsorbed ZDDP and peak (b): zinc phosphate. (d) S k-edge normalized heights of peak (e): alkyl sulfide and zinc sulfide, peak (f): alkyl disulfide and peak (g): zinc sulfate. Each curve in (a) and (b) is vertically offset for clarity by adding a constant, as specified on each curve, relative to the first curve.

did not appear to have an initial induction period. This is evident from the decrease in the area of peak (a) of adsorbed ZDDP molecules to the steel surface and simultaneous increase in the area of peak (b) of the zinc phosphate without time lag, as shown in Fig. 6c. During this decomposition phase, the zinc phosphate is formed at a rate sixfold and threefold slower than the one of the tribofilms formed at high temperature of $80{ }^{\circ} \mathrm{C}$ and 2.2 and $1.0 \mathrm{GPa}$, respectively, but still sixfold faster than the rate of the thermal film at the same temperature but without shear. This confirms that the ZDDP decomposition and tribofilm formation can be accelerated by both shear and heat.

As shearing continued, a slow increase in the area of the adsorbed ZDDP, peak (a), occurred accompanied by a slow decrease in the area of the zinc phosphate, peak (b), at a similar rate. This trend is in contrast to the ones observed in the case of the tribo- and thermal films formed at high temperature. One possible explanation for this slower and overall less growth of zinc phosphate (e.g. area of peak (b) in Fig. $6 \mathrm{c}$ is less than $2 \mathrm{eV}$ compared to about $6 \mathrm{eV}$ in Fig. $3 \mathrm{c}$ and $4 \mathrm{eV}$ in Fig. 5c) can be related to the low tenacity and minuscule amount of the formed phosphate film at low temperature. The low tenacious tribofilm can be removed easily, which exposes the steel surface underneath. The newly available steel surface can then be covered by more adsorbed ZDDP. Thus, the adsorbed ZDDP area appears to increase as shearing continues. Furthermore, this may suggest that the decomposition of the adsorbed ZDDP at low temperature occurs slowly and immaturely. Therefore, within the time of experiments, plenty of adsorbed ZDDP molecules are still present on the surface.

\section{ZDDP tribofilms formed at $80{ }^{\circ} \mathrm{C}$ and $1.0 \mathrm{GPa}$ : DLC coated countersurfaces}

The evolution of the $\mathrm{P}$ k-edge spectra inside the wear scar formed between DLC coated countersurfaces is shown in Fig. 7. Similar to the previously studied fast evolving tribofilms formed at high temperature and contact pressure on bare steel surfaces, shearing seemed to induce the decomposition of the adsorbed ZDDP to form zinc phosphate immediately after the tribotest started without any observed induction period. The evolution of the P k-edge peaks shown in Fig. $7 \mathrm{c}$ indicates that the first 
(a)

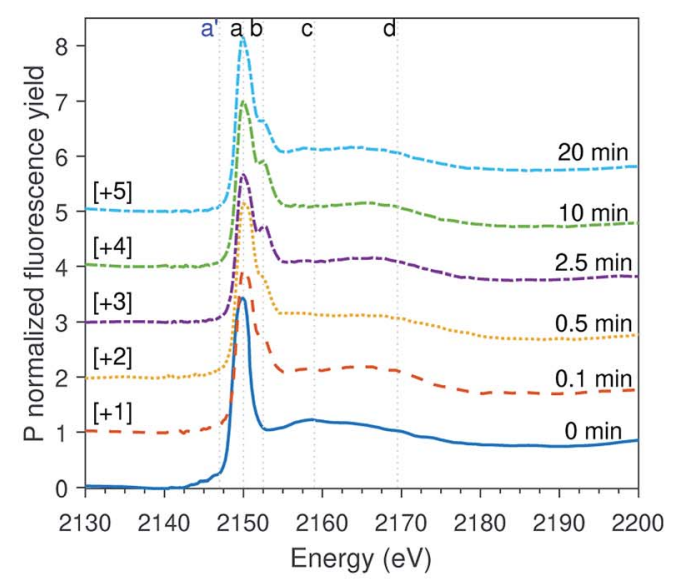

(b)

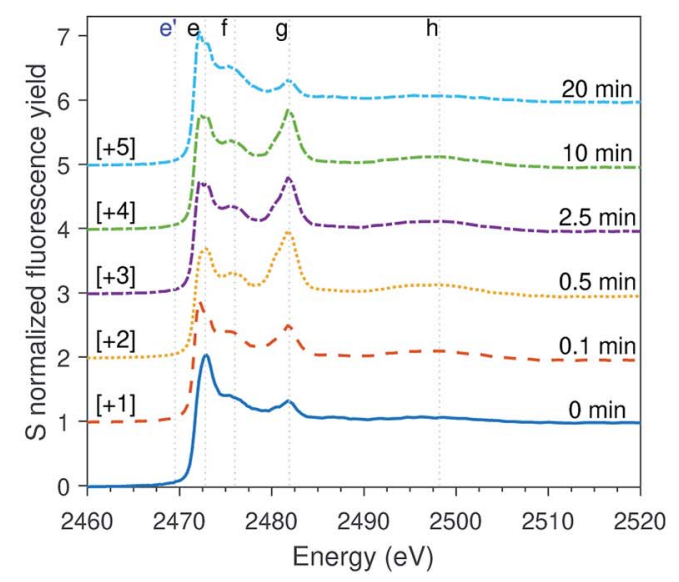

(c)

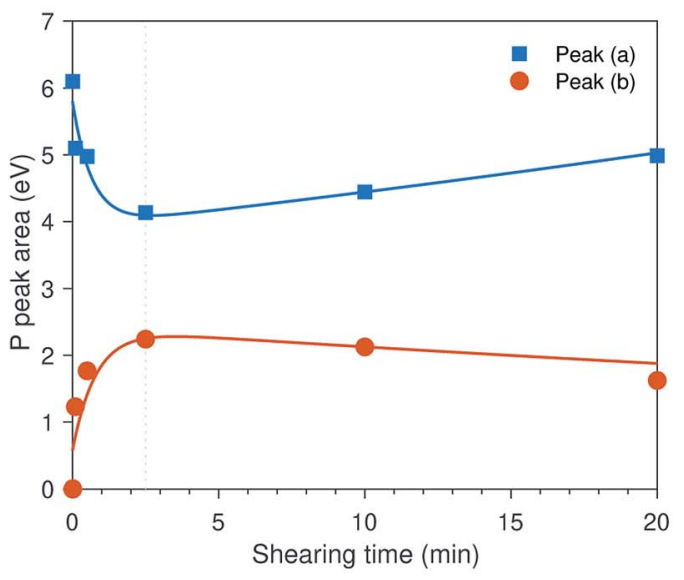

(d)

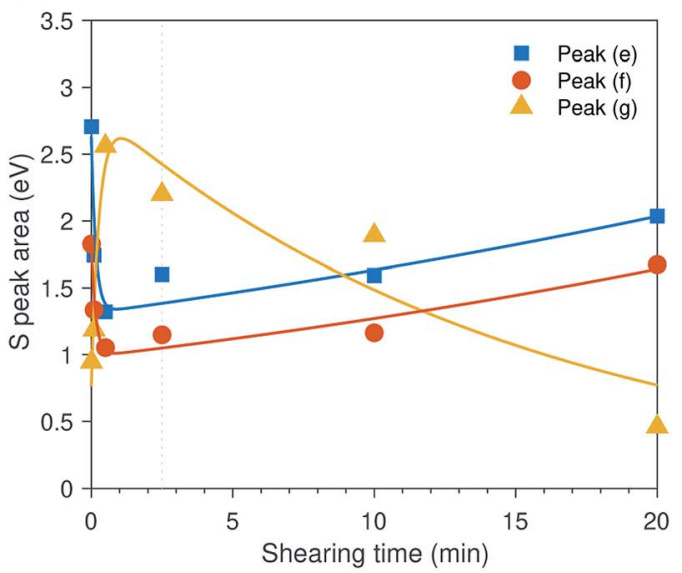

Fig. 6 Spectra evolution of the ZDDP normalized fluorescence yield after different shearing times at $25^{\circ} \mathrm{C}$ and $1 \mathrm{GPa}$. (a) and (b) Spectra of $\mathrm{P}$ and $\mathrm{S}$ k-edges, respectively. (c) P k-edge normalized heights of peak (a): adsorbed ZDDP and peak (b): zinc phosphate. (d) S k-edge normalized heights of peak (e): alkyl sulfide and zinc sulfide, peak (f): alkyl disulfide and peak (g): zinc sulfate. Each curve in (a) and (b) is vertically offset for clarity by adding a constant, as specified on each curve, relative to the first curve.

decomposition phase started with the fast decrease in the area of the adsorbed ZDDP, peak (a), i.e. marking the outset of the ZDDP decomposition, accompanied by the fast increase in the area of the zinc phosphate, peak (b), i.e. indicating the formation of a tribofilm that contains zinc phosphate whether of short or long chains. This occurs at a fast rate similar to the one of the tribofilm formed on bare metal surfaces at high temperature and contact pressure but at least tenfold faster than the rate of the thermal film or the tribofilm formed at low temperature of $25{ }^{\circ} \mathrm{C}$. Furthermore, during this phase, the sulfur composing the tribofilm appeared mainly in the oxidized zinc sulfate form in addition to the reduced zinc sulfide form.

After longer shearing time, the zinc sulfate concentration decreased whereas the concentration of alkyl sulfide and zinc sulfide: peak (e) and alkyl disulfide: peak (f) increased. Moreover, a negligible decrease occurred in the areas of the adsorbed ZDDP, peak (a), and zinc phosphate, peak (b). One possible explanation for this trend can be related to the low durability of the formed tribofilm on the DLC surface causing the tribofilm to be removed easily. The results are in line with several previous studies, which reported that the P-based additives such as ZDDP can react and form thin tribofilms on DLC coatings even without containing any doped cations. ${ }^{43,44,46,47,49}$ In contrast, other studies found that no tribofilms can be formed on non-doped DLC coatings without metallic cations. ${ }^{37,40-42}$ The main factor behind the wide disparity between the formation and absence of tribofilms on DLC coatings appears to be related to the tribofilm tenacity as discussed earlier. Several studies ${ }^{43,44}$ have already suggested that the tribofilms formed on DLC-DLC contacting surfaces are less tenacious than the ones formed on metal surfaces. Furthermore, no iron was present in the formed tribofilm on DLC surfaces, which suggests that the low tenacity to the coating surface can be related to the absence of mixed oxide/sulfide base layer. This layer seems to act as a glue joining the substrate with the subsequently formed phosphate layers composing the main bulk of the tribofilm. It follows that any formed tribofilms on DLC coatings, especially if non-doped, will weakly adhere to the coating leading to its effortless removal under rubbing once formed. 
(a)

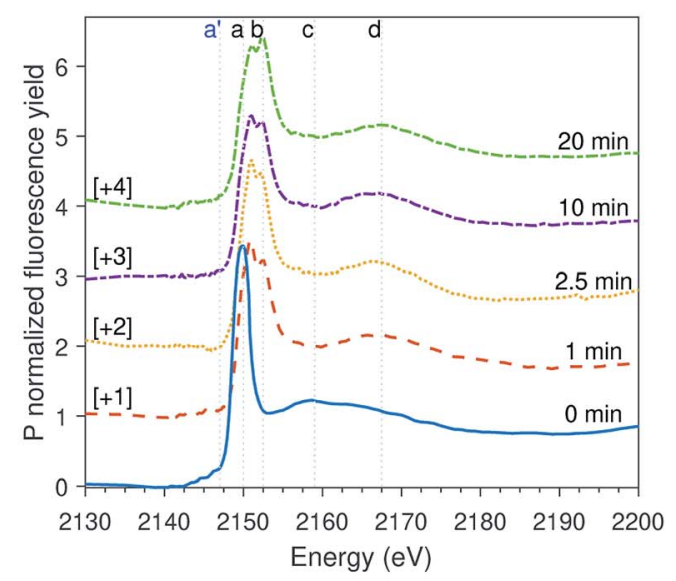

(b)

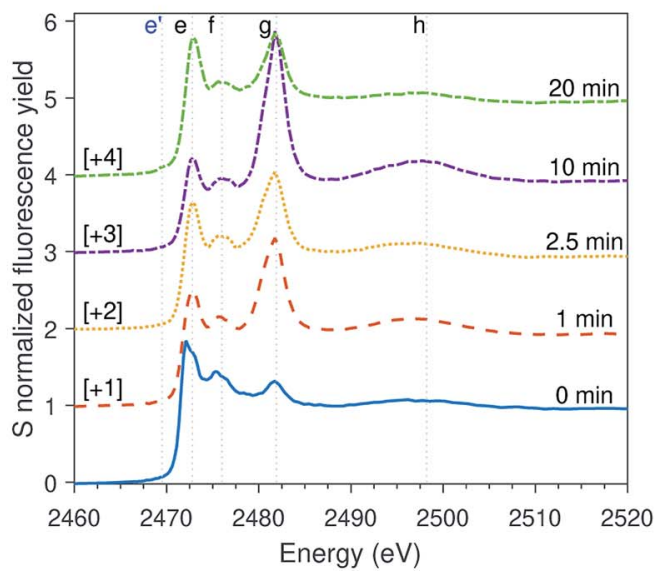

(c)

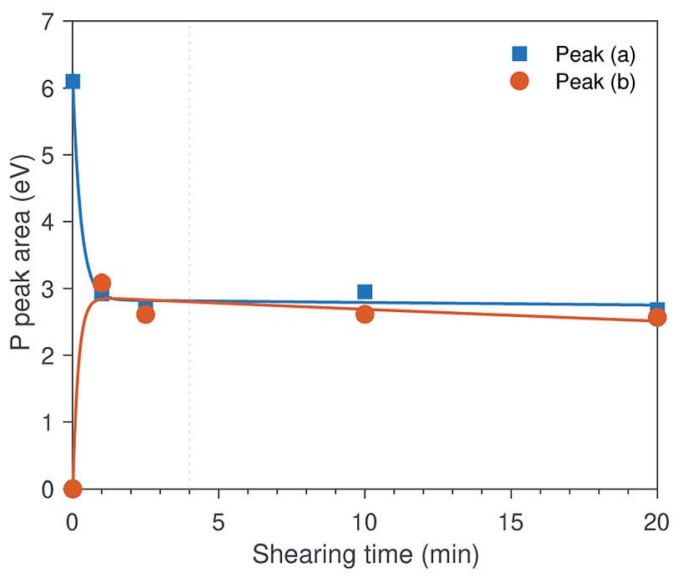

(d)

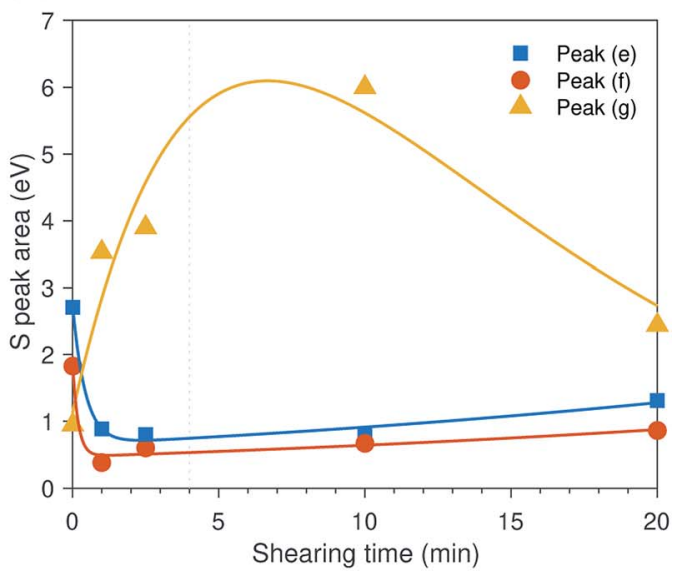

Fig. 7 Spectra evolution of the ZDDP normalized fluorescence yield after different shearing times at $80{ }^{\circ} \mathrm{C}$ and 1 GPa using DLC coated countersurfaces. (a) and (b) Spectra of P and S k-edges, respectively. (c) P k-edge normalized heights of peak (a): adsorbed ZDDP and peak (b): zinc phosphate. (d) S k-edge normalized heights of peak (e): alkyl sulfide and zinc sulfide, peak (f): alkyl disulfide and peak (g): zinc sulfate. Each curve in (a) and (b) is vertically offset for clarity by adding a constant, as specified on each curve, relative to the first curve.

\section{Overall discussion}

In the previous sections, each tribo- or thermal test was discussed individually. These tests were performed under different operating conditions of temperature, contact pressure and type of countersurfaces. To understand the effect of these conditions on the formed tribo- and thermal films, it is necessary to compare the formed P- and S-species across the different tests.

Fig. 8a compares the normalized areas of the adsorbed ZDDP, peak (a), and zinc phosphate, peak (b), at the P k-edge of the ZDDP tribo- and thermal films at the end of each test. The available data exhibit three distinctive features. First, as the operating conditions become harsher, i.e. higher temperature and contact pressure, or just longer heating or shearing time, the area of the adsorbed ZDDP peak decreases accompanied by a general increase in the area of the zinc phosphate peak.

The significance of this observation is that it confirms that heat and shear role is just to accelerate the reaction kinetics of the additive decomposition, but they do not affect the type of the formed species. For instance, observing the areas of the adsorbed ZDDP and zinc phosphate peaks of the tribofilm formed under $2.2 \mathrm{GPa}$ at $80{ }^{\circ} \mathrm{C}$ after $60 \mathrm{~min}$ and the ones of the thermal film formed under heating for 24 hours at $80{ }^{\circ} \mathrm{C}$ confirms that despite the different kinetics governing the formation of these tribo- and thermal films, their final composition appears to be similar, which is in agreement with several previous studies. ${ }^{7,10,16,24}$ The ZDDP can decompose thermally and under rubbing to form films consisting mainly of a large content of phosphate bonds, e.g. $\mathrm{P}-\mathrm{O}-, \mathrm{P}=\mathrm{O}$ and $\mathrm{P}-\mathrm{O}-$ P. ${ }^{61}$

Second, there is an upper limit for the area of the zinc phosphate peak, which is reached after long rubbing or heating time. Beyond this point, either the zinc phosphate area reaches steady state or starts to decrease. This is evident from the maximum zinc phosphate peak area reached in the tests performed at $2.2 \mathrm{GPa}$ after 20 and 60 minutes of rubbing. This observation is also in agreement with the findings of each test individually. The evolution of the normalized area of the zinc phosphate peak can be linked to evolution of the formed zinc phosphate chains. The chain length is typically a complex function of the operating conditions, ${ }^{24}$ e.g. load and temperature, as well as the oil additives, dispersant and contaminants 
(a)

Fig. 8 Comparison between the normalized areas of the main peaks at the (a) $\mathrm{P}$ k-edge and (b) $\mathrm{S}$ k-edge of the ZDDP tribo- and thermal films at the end of each in situ test. All the test were performed using steel-steel countersurfaces except the one indicated by the arrow in which DLC-DLC countersurfaces were used.

such as water. ${ }^{13}$ The overall data suggest that by increasing the heating or rubbing time, zinc phosphate chains are polymerized to form longer chains of zinc poly-phosphate, which can reach steady state length or be shortened with the progression of heating or rubbing. The eventual decrease in chain length can be related to different factors, which include (i) the scission of long zinc phosphate chains into shorter ones under the large contact pressure, (ii) severe surface wear that can remove part of the long chains and (iii) the depolymerization reaction occurring to the long chains to form short zinc ortho- or pyrophosphate chains especially near the metal surface. ${ }^{18-21}$ The depolymerization can occur due to the reaction of the initially formed zinc phosphate chains with metal oxides, e.g. $\mathrm{ZnO}, \mathrm{FeO}$ or $\mathrm{Fe}_{2} \mathrm{O}_{3}$, near the substrate, which can cleave any formed long chains into shorter ones of mixed Fe-Zn or Fe phosphates. ${ }^{18-20}$

Third, there seems to be a trend that the larger the area of the zinc phosphate peak, the smaller the area of the adsorbed ZDDP peak. As discussed before, this can be related to the availability of the substrate, whether bare or DLC coated steel surface. As more tribofilm volume is formed under shearing or heating, less substrate becomes available for the additive adsorption. This may indicate that the ZDDP molecules do not adsorb preferentially to the tribofilm and thus the detected signal comes mainly from the entrapped additive within the formed tribofilm itself and to a less extent from its upper surface.

Examining the sulfur-based species formed under the various tested operating conditions can provide a better understanding of the composition evolution of the ZDDP triboand thermal films. Fig. 8b compares the normalized areas of the alkyl sulfide and ZnS, peak (e), and zinc sulfate, peak (g), at the S k-edge of the ZDDP films at the end of each test. As the adsorbed ZDDP molecules continuously decompose over rubbing or heating time, peak (e) is expected to evolve such that the concentration of alkyl sulfide is continuously decreasing whereas the one of $\mathrm{ZnS}$ is continuously increasing. Thus, over time, peak (e) is expected to represent more $\mathrm{ZnS}$ than alkyl sulfide from the adsorbed ZDDP.

Similar to the P k-edge data, the available S data exhibit several distinctive features. First, as the temperature and contact pressure increase or just the heating or shearing time gets longer, the area of the alkyl sulfide and $\mathrm{ZnS}$ peak initially decreased whereas the area of the zinc sulfate peak increased. However, as the temperature, contact pressure, shearing or heating time increases the trend eventually reverses, i.e. alkyl sulfide and $\mathrm{ZnS}$ increased and zinc sulfate decreased. The overall trend across the various tribo- and thermal films is strikingly similar to the one observed in the evolution over time of the individual tribo- and thermal films, which confirms that the observed trend across the various tests is just related to the difference in the reaction kinetics. Throughout the ZDDP decomposition, sulfur starts from alkyl sulfide from adsorbed ZDDP and ends with zinc sulfide. Therefore, the apparent decrease and then increase in the zinc sulfide area could mean that the initial sulfide area consists mainly of alkyl sulfide from the adsorbed ZDDP whereas the final area consists primarily of zinc sulfide and a small concentration of alkyl sulfide from the adsorbed ZDDP embedded within the tribofilm.

The second feature in the data is related to the reduction of zinc sulfate as the operating conditions get harsher. Under mild conditions, the zinc sulfate area starts to increase, which reaches a peak value before starts to decrease to a minuscule value under harsher conditions or longer tests. These changes are mainly related to the potent effect of both rubbing and heat, which can accelerate the decomposition of the adsorbed ZDDP and formation of tribofilm consisting mainly of zinc phosphate following the reduction of zinc sulfate to zinc sulfide. 


\section{Conclusion}

A mini pin-on-disc tribotester was designed, which can be coupled with XAS to perform in situ tribological tests at different contact pressures, temperatures and sliding speeds while examining the composition evolution over time of the formed tribo- and thermal films. The main in situ XAS results together with the findings from earlier studies suggest that the ZDDP decomposition process occurs as follows:

(i) The unreacted ZDDP adsorbs to the substrate, whether a bare steel or DLC coated surface in the form of a rearranged linkage isomer in which the alkyl groups have migrated from $\mathrm{O}$ to $\mathrm{S}$ atoms.

(ii) The adsorbed molecules decompose partially by losing sulfur to form zinc sulfate species.

(iii) The formed zinc sulfate is reduced to form zinc sulfide.

(iv) The adsorbed molecules decompose completely to form zinc phosphate of chains progressively increasing in length.

(v) Any harsh conditions, i.e. high temperature and large contact pressure, or even just long shearing or heating time can cleave the long zinc phosphate chains into shorter ones.

(vi) Once the tribofilm consisting mainly of zinc phosphate covers the substrate completely, the ZDDP molecules do not seem to adsorb preferentially to the tribo- or thermal film covering the substrate. The origin of the detected adsorbed molecules in the late stages of the test is mainly the trapped additive within the formed film, which occurs during the early stages of the test or mechanically mixed without significant replenishment.

(vii) The formed tribofilm on the DLC coated surface or at low temperature, i.e. $25{ }^{\circ} \mathrm{C}$, is of small volume and low tenacity. The low tenacious tribofilm was related to the absence of mixed oxide/sulfide base layer. This layer seems to act as a glue joining the substrate with the subsequently formed zinc phosphate layers composing the main bulk of the tribofilm.

The findings of this study advance our understanding of the origin of the antiwear properties of the ZDDP triboreactive layers, which can significantly contribute towards finding green alternatives. Furthermore, the employed methodologies and findings of this study widen the scope of the future investigations to characterize various triboreactive materials and follow their reaction kinetics under shear, temperature and load in situ as they evolve.

\section{Conflicts of interest}

There are no conflicts to declare.

\section{Acknowledgements}

This work is supported by EPSRC (grant number EP/R001766/1), Marie Curie Initial Training Networks (grant number 317334) and Diamond Light Source (proposal no. SP12907, beamline I18).

\section{References}

1 A. M. Barnes, K. D. Bartle and V. R. Thibon, A review of zinc dialkyldithiophosphates (ZDDPS): characterisation and role in the lubricating oil, Tribol. Int., 2001, 34, 389-395.

2 H. Spikes, The History and Mechanisms of ZDDP, Tribol. Lett., 2004, 17, 469-489.

3 M. a. Nicholls, T. Do, P. R. Norton, M. Kasrai and G. Bancroft, Review of the lubrication of metallic surfaces by zinc dialkyldithiophosphates, Tribol. Int., 2005, 38, 15-39.

4 API, Engine Oil Licensing and Certification System, tech. rep. API 1509, American Petroleum Institute, 2014, pp. 1-138.

5 J. Andersson, M. Antonsson, L. Eurenius, E. Olsson and M. Skoglundh, Deactivation of diesel oxidation catalysts: vehicle- and synthetic aging correlations, Appl. Catal., B, 2007, 72, 71-81.

6 C. Larese, F. C. Galisteo, M. L. Granados, R. Mariscal, J. Fierro, M. Furió and R. F. Ruiz, Deactivation of real three way catalysts by CePO 4 formation, Appl. Catal., B, 2003, 40, 305-317.

7 M. Nicholls, M. Najman, Z. Zhang, M. Kasrai, P. Norton and P. Gilbert, The contribution of XANES spectroscopy to tribology, Can. J. Chem., 2007, 85, 816-830.

8 N. Lindsay, R. Carter III, P. Schmitz, L. Haack, R. Chase, J. deVries and P. Willermet, Characterization of films formed at a lubricated cam/tappet contact, Spectrochim. Acta, Part A, 1993, 49, 2057-2070.

9 G. C. Smith, Evaluation of a simple correction for the hydrocarbon contamination layer in quantitative surface analysis by XPS, J. Electron Spectrosc. Relat. Phenom., 2005, 148, 21-28.

10 A. Morina, H. Zhao and J. F. W. Mosselmans, In situ reflection-XANES study of ZDDP and MoDTC lubricant films formed on steel and diamond like carbon (DLC) surfaces, Appl. Surf. Sci., 2014, 297, 167-175.

11 I. Nedelcu, E. Piras, A. Rossi and H. Pasaribu, XPS analysis on the influence of water on the evolution of zinc dialkyldithiophosphate-derived reaction layer in lubricated rolling contacts, Surf. Interface Anal., 2012, 44, 1219-1224.

12 H. Cen, A. Morina, A. Neville, R. Pasaribu and I. Nedelcu, Effect of water on ZDDP anti-wear performance and related tribochemistry in lubricated steel/steel pure sliding contacts, Tribol. Int., 2012, 56, 47-57.

13 P. Parsaeian, M. C. Van Eijk, I. Nedelcu, A. Neville and A. Morina, Study of the interfacial mechanism of ZDDP tribofilm in humid environment and its effect on tribochemical wear; part I: experimental, Tribol. Int., 2017, 107, 135-143.

14 A. Dorgham, A. Neville, K. Ignatyev, F. Mosselmans and A. Morina, An in situ synchrotron XAS methodology for surface analysis under high temperature, pressure, and shear, Rev. Sci. Instrum., 2017, 88, 015101.

15 A. Ciniero, J. Le Rouzic, I. Baikie and T. Reddyhoff, The origins of triboemission-correlating wear damage with electron emission, Wear, 2017, 374, 113-119. 
16 Z. Zhang, E. Yamaguchi, M. Kasrai, G. Bancroft, X. Liu and M. Fleet, Tribofilms generated from ZDDP and DDP on steel surfaces: part 2, chemistry, Tribol. Lett., 2005, 19, 221-229.

17 N. Gosvami, J. Bares, F. Mangolini, A. Konicek, D. Yablon and R. Carpick, Mechanisms of antiwear tribofilm growth revealed in situ by single-asperity sliding contacts, Science, 2015, 348, 102-106.

18 M. Crobu, A. Rossi, F. Mangolini and N. D. Spencer, Tribochemistry of bulk zinc metaphosphate glasses, Tribol. Lett., 2010, 39, 121-134.

19 J. M. Martin, Antiwear mechanisms of zinc dithiophosphate: a chemical hardness approach, Tribol. Lett., 1999, 6, 1-8.

20 J. M. Martin, C. Grossiord, T. Le Mogne, S. Bec and A. Tonck, The two-layer structure of Zndtp tribofilms: part I: AES, XPS and XANES analyses, Tribol. Int., 2001, 34, 523-530.

21 R. Heuberger, A. Rossi and N. D. Spencer, Pressure dependence of ZnDTP tribochemical film formation: a combinatorial approach, Tribol. Lett., 2007, 28, 209-222.

22 R. Heuberger, A. Rossi and N. D. Spencer, XPS study of the influence of temperature on ZnDTP tribofilm composition, Tribol. Lett., 2007, 25, 185-196.

23 R. Mourhatch and P. B. Aswath, Tribological behavior and nature of tribofilms generated from fluorinated ZDDP in comparison to ZDDP under extreme pressure conditionspart 1: structure and chemistry of tribofilms, Tribol. Int., 2011, 44, 187-200.

24 Z. Yin, M. Kasrai, M. Fuller, G. M. Bancroft, K. Fyfe and K. H. Tan, Application of soft X-ray absorption spectroscopy in chemical characterization of antiwear films generated by ZDDP part I: the effects of physical parameters, Wear, 1997, 202, 172-191.

25 B. Kim, R. Mourhatch and P. B. Aswath, Properties of tribofilms formed with ashless dithiophosphate and zinc dialkyl dithiophosphate under extreme pressure conditions, Wear, 2010, 268, 579-591.

26 E. Ferrari, K. Roberts, M. Sansone and D. Adams, A multiedge X-ray absorption spectroscopy study of the reactivity of zinc di-alkyl-di-thiophosphates anti-wear additives: 2 . In situ studies of steel/oil interfaces, Wear, 1999, 236, 259-275.

27 J. F. W. Mosselmans, P. D. Quinn, A. J. Dent, S. A. Cavill, S. D. Moreno, A. Peach, P. J. Leicester, S. J. Keylock, S. R. Gregory, K. D. Atkinson, et al., I18 - the microfocus spectroscopy beamline at the diamond light source, $J$. Synchrotron Radiat., 2009, 16, 818-824.

28 G. Das, M. Tiwari, A. Singh and H. Ghosh, Effect of synchrotron polarization on grazing incidence X-ray fluorescence analysis, J. Anal. At. Spectrom., 2014, 29, 24052413.

29 B. Ravel and M. Newville, ATHENA and ARTEMIS: interactive graphical data analysis using IFEFFIT, Phys. Scr., 2005, 2005, 1007.

30 G. S. Henderson, F. M. De Groot and B. J. Moulton, X-ray absorption near-edge structure (XANES) spectroscopy, Rev. Mineral. Geochem., 2014, 78, 75-138.

31 J. Benedet, J. H. Green, G. D. Lamb and H. A. Spikes, Spurious mild wear measurement using white light interference microscopy in the presence of antiwear films, Tribol. Trans., 2009, 52, 841-846.

32 K. Topolovec-Miklozic, T. R. Forbus and H. A. Spikes, Film thickness and roughness of ZDDP antiwear films, Tribol. Lett., 2007, 26, 161-171.

33 L. Taylor, A. Dratva and H. Spikes, Friction and wear behavior of zinc dialkyldithiophosphate additive, Tribol. Trans., 2000, 43, 469-479.

34 L. J. Taylor and H. A. Spikes, Friction-Enhancing Properties of ZDDP Antiwear Additive: Part I- Friction and Morphology of ZDDP Reaction Films, Tribol. Trans., 2003, 46, 303-309.

35 L. J. Taylor and H. A. Spikes, Friction-enhancing properties of ZDDP antiwear additive: part II- influence of ZDDP reaction films on EHD lubrication, Tribol. Trans., 2003, 46, 310-314.

36 N. N. Gosvami, P. C. Nalam, A. L. Exarhos, Q. Tam, J. M. Kikkawa and R. W. Carpick, Direct torsional actuation of microcantilevers using magnetic excitation, Appl. Phys. Lett., 2014, 105, 093101.

37 T. Haque, A. Morina, A. Neville, R. Kapadia and S. Arrowsmith, Non-ferrous coating/lubricant interactions in tribological contacts: assessment of tribofilms, Tribol. Int., 2007, 40, 1603-1612.

38 B. Podgornik, S. Jacobson and S. Hogmark, DLC coating of boundary lubricated components - advantages of coating one of the contact surfaces rather than both or none, Tribol. Int., 2003, 36, 843-849.

39 B. Podgornik, J. Vižintin, S. Jacobson and S. Hogmark, Tribological behaviour of WC/C coatings operating under different lubrication regimes, Surf. Coat. Technol., 2004, 177, 558-565.

40 B. Podgornik and J. Vižintin, Tribological reactions between oil additives and DLC coatings for automotive applications, Surf. Coat. Technol., 2005, 200, 1982-1989.

41 M. de Barros' Bouchet, J. Martin, T. Le-Mogne and B. Vacher, Boundary lubrication mechanisms of carbon coatings by MoDTC and ZDDP additives, Tribol. Int., 2005, 38, 257-264.

42 M. Ban, M. Ryoji, S. Fujii and J. Fujioka, Tribological characteristics of Si-containing diamond-like carbon films under oil-lubrication, Wear, 2002, 253, 331-338.

43 B. Vengudusamy, J. H. Green, G. D. Lamb and H. A. Spikes, Tribological properties of tribofilms formed from ZDDP in DLC/DLC and DLC/steel contacts, Tribol. Int., 2011, 44, 165-174.

44 S. Equey, S. Roos, U. Mueller, R. Hauert, N. D. Spencer and R. Crockett, Tribofilm formation from ZnDTP on diamondlike carbon, Wear, 2008, 264, 316-321.

45 S. Equey, S. Roos, U. Mueller, R. Hauert, N. D. Spencer and R. Crockett, Reactions of zinc-free antiwear additives in DLC/DLC and steel/steel contacts, Tribol. Int., 2008, 41, 1090-1096.

46 K. Topolovec-Miklozic, F. Lockwood and H. Spikes, Behaviour of boundary lubricating additives on DLC coatings, Wear, 2008, 265, 1893-1901.

47 M. Kalin, E. Roman, L. Ožbolt and J. Vižintin, Metaldoped (Ti, WC) diamond-like-carbon coatings: reactions with 
extreme-pressure oil additives under tribological and static conditions, Thin Solid Films, 2010, 518, 4336-4344.

48 M. Kalin, E. Roman and J. Vižintin, The effect of temperature on the tribological mechanisms and reactivity of hydrogenated, amorphous diamond-like carbon coatings under oil-lubricated conditions, Thin Solid Films, 2007, 515, 3644-3652.

49 S. Akbari, J. Kovač and M. Kalin, Effect of ZDDP concentration on the thermal film formation on steel, hydrogenated non-doped and Si-doped DLC, Appl. Surf. Sci., 2016, 383, 191-199.

50 M. Najman, M. Kasrai and G. Bancroft, Chemistry of antiwear films from ashless thiophosphate oil additives, Tribol. Lett., 2004, 17, 217-229.

$51 \mathrm{R}$. Jones and R. Coy, The chemistry of the thermal degradation of zinc dialkyldithiophosphate additives, ASLE Trans., 1981, 24, 91-97.

52 M. L. S. Fuller, M. Kasrai, G. M. Bancroft, K. Fyfe and K. H. Tan, Solution decomposition of zinc dialkyl dithiophosphate and its effect on antiwear and thermal film formation studied by X-ray absorption spectroscopy, Tribol. Int., 1998, 31, 627-644.

53 K. Masenelli-Varlot, M. Kasrai, G. Bancroft, G. De Stasio, B. Gilbert, E. Yamaguchi and P. Ryason, Spatial distribution of the chemical species generated under rubbing from ZDDP and dispersed potassium triborate, Tribol. Lett., 2003, 14, 157-166.

54 Z. Zhang, M. Kasrai, G. Bancroft and E. Yamaguchi, Study of the interaction of ZDDP and dispersants using X-ray absorption near edge structure spectroscopy - part 1: thermal chemical reactions, Tribol. Lett., 2003, 15, 377-384.

55 M. Newville, Fundamentals of XAFS, Rev. Mineral. Geochem., 2014, 78, 33-74.

56 M. Najman, M. Kasrai and G. Bancroft, X-ray absorption spectroscopy and atomic force microscopy of films generated from organosulfur extreme-pressure (EP) oil additives, Tribol. Lett., 2003, 14, 225-235.

57 X. Chen, P. B. Aswath and R. L. Elsenbaumer, US Pat. 8,791,056, 2014.

58 B. Brendebach, M. Denecke, G. Roth and S. Weisenburger, Sulfur incorporation in high level nuclear waste glass: AS K-edge XAFS investigation, J. Phys.: Conf. Ser., 2009, 190, 012186.

59 B. Kim, V. Sharma and P. B. Aswath, Chemical and mechanistic interpretation of thermal films formed by dithiophosphates using XANES, Tribol. Int., 2017, 114, 1526.

60 G. Pereira, D. Munoz-Paniagua, A. Lachenwitzer, M. Kasrai, P. R. Norton, T. W. Capehart, T. A. Perry and Y.-T. Cheng, A variable temperature mechanical analysis of ZDDPderived antiwear films formed on 52100 steel, Wear, 2007, 262, 461-470.

61 M. Crobu, A. Rossi, F. Mangolini and N. D. Spencer, Chainlength-identification strategy in zinc polyphosphate glasses by means of XPS and ToFSIMS, Anal. Bioanal. Chem., 2012, 403, 1415-1432. 